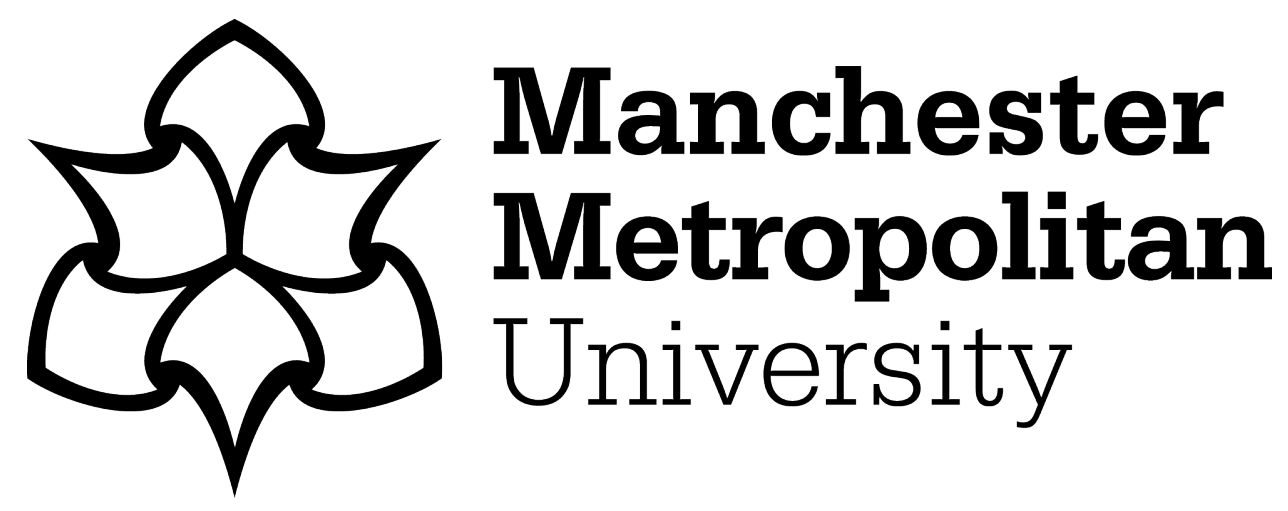

Down, Michael, Rowley-Neale, SJ, Smith, GC and Banks, Craig (2018) Fabrication of Graphene Oxide Supercapacitor Devices. ACS Applied Energy Materials, 1 (2). pp. 707-714. ISSN 2574-0962

Downloaded from: https://e-space.mmu.ac.uk/621273/

Version: Accepted Version

Publisher: ACS

DOI: https://doi.org/10.1021/acsaem.7b00164

Please cite the published version 


\section{Fabrication of Graphene Oxide Supercapacitor Devices}

Michael P. Down, ${ }^{1,2}$ Samuel J. Rowley-Neale, ${ }^{1,2}$ Graham C. Smith ${ }^{3}$ and Craig E. Banks ${ }^{1,2 *}$

${ }^{1}$ :Faculty of Science and Engineering, Manchester Metropolitan University, Chester Street, Manchester, M1 5GD, UK

${ }^{2}$ :Manchester Fuel Cell Innovation Centre, Manchester Metropolitan University, Chester Street, Manchester M1 5GD, UK.

${ }^{3}$ : Faculty of Science and Engineering, Department of Natural Sciences, University of Chester, Thornton Science Park, Pool Lane, Ince, Chester CH2 4NU, UK.

\footnotetext{
*To whom correspondence should be addressed.

Email: c.banks@mmu.ac.uk; Tel: ++(0)1612471196; Fax: ++(0)1612476831

Website: www.craigbanksresearch.com
} 


\begin{abstract}
The fabrication, characterisation and energy storage capacity of a graphene oxide (GO) based supercapacitor device is reported. This device is fabricated via a facile screen-printing technique, providing a highly reproducible and flexible symmetrical supercapacitor device. The capacitive properties of these GO devices are investigated in both aqueous electrolytes and room temperature ionic liquids. They are shown to improve the capacitive performance from $0.82 \mathrm{~F} \mathrm{~g}^{-1}$ displayed by a graphitic screen, to $423 \mathrm{Fg}^{-1}$ representing a $\mathrm{ca}$. 500 fold increase. The GO supercapacitor device exhibits a highly competitive capacitance of $423 \mathrm{Fg}^{-1}$, with a impressive power handling capability of up to $13.9 \mathrm{~kW} \mathrm{~kg}^{-1}$ and an energy density of $11.6 \mathrm{Wh}$ $\mathrm{kg}^{-1}$. This work demonstrates that GO, before it has been reduced to graphene, is a high performance supercapacitor material of its own rite.
\end{abstract}

Keywords: Graphene Oxide, Supercapacitor, Capacitance, Sustainable, Screen-Printing 


\section{Broader Context}

There is a demand within the global energy economy to seek out new less polluting alternatives to the current energy generation / storage techniques, which currently rely predominately upon fossil fuels. Supercapacitors are electrochemical energy storage systems that are finding applications in many technological fields. Whilst, the energy density of the supercapacitor is much higher than that of a conventional dielectric capacitor, it is still lower than that of batteries and fuel cells. The evolution of nanostructured materials has offered opportunities for the development of high-energy density supercapacitors. Despite this, nanomaterials are predominantly characterised as deposited on an existing electrode material as an additive or enhancement, or on a substrate, which provides the mechanical support required for the nanomaterial. Consequently, the development of an energy storage platform that applies such advanced developed materials is greatly hindered by the requirement of integrating the substrate into the final device specification and manufacture. Here, we demonstrate an approach to enhancing the performance of supercapacitors. A graphene oxide based supercapacitor device is rapidly fabricated by screen-printing technology, exhibiting impressive specific capacitance and energy storage properties. A screen-printing ink is enhanced with the integration of functionalised graphene to provide a contextual analysis of the impact of graphene oxide in capacitor electrodes. The present work will generate a broad range of interest for those who work on graphene-based materials and energy storage devices. 


\section{Introduction}

Electrochemical capacitors, or supercapacitors, are passive and static electrical storage devices, which exhibit rapid charging rates. The intrinsic properties associated with typical supercapacitors are high power capabilities, fast charge propagation, charge-discharge process occurring within seconds, long cyclic life, low maintenance, and low self-discharging. ${ }^{1-5}$ Supercapacitors also exhibit larger energy density compared to conventional capacitors, resulting in a device that provides the capability of charging quicker and storing more energy. As a result, significant interest has developed around the field of energy storage in supercapacitors and supercapacitor-battery hybrid devices with research focusing on the development of novel materials that could beneficial increase the efficiency of these devices.

Graphene, due to its two-dimensional (2D) crystalline structure, exhibits interesting electronic, optical and mechanical properties due to its two-dimensional (2D) crystalline structure. The charge carriers of graphene, i.e. the electrons, move according to ballistic mechanism in the plane of the crystal lattice, hence the material exhibits high conductivity despite it being an organic compound. ${ }^{6}$ With this crystalline structure, graphene is a building block for the majority of carbon based materials because it can be wrapped up to form a buckminsterfullerene, rolled to form a carbon nanotube, ${ }^{7}$ grown on cellular macrostructures to generate freestanding $3 \mathrm{D}$ graphene macrostructures ${ }^{8}$ or quite simply layered to form graphite. ${ }^{9}$ Each of these variations form materials with wildly variable electronic properties. Graphene can be produced by mechanical exfoliation ${ }^{10}$ however the quantities produced by this method are small and suitable for individual research purposes only. Therefore, an alternative method is required to mass-produce graphene. One alternative is by means of chemical oxidation. The resulting graphene oxide (GO) is typically reduced to graphene and used as the basis of the energy storage devices. ${ }^{11}$

An essential criterion expected for energy storage devices is its longevity and stability in spite of extensive usage and fast charging rate during recharging. Producing an energy storage device, which exhibits these traits is not easily achievable in common battery arrangements. Therefore, supercapacitors, which demonstrate higher power handling capabilities and can implement the high charge rates, are incorporated 'inside' or in parallel with the battery to comply with this criteria. In order to meet these requirements, supercapacitors are typically made of porous carbon but recently, the researchers have started to investigate the possibilities of using graphene as electrodes. For instance the theoretical areal capacitance is $\sim 21 \mu \mathrm{F} \mathrm{cm}{ }^{-2}$, 
which equates to roughly $\sim 550 \mathrm{~F} \mathrm{~g} \mathrm{~g}^{-1}$ when the entire surface is utilised. ${ }^{12}$ However, the practical capacitive behaviour of pure graphene is lower than the anticipated value due to the serious agglomeration during both the preparation and application processes. Therefore, boosting the overall electrochemical performance of graphene-based materials remains a great challenge. As a result, solutions that are more exotic are frequently investigated, and given the additional step required in the manufacturing process to produce graphene from its oxidised counterpart there is increased interest in in utilising GO in energy storage applications. For example, a process of co-reduction to reduce dispersed GO and single-walled carbon nanotubes (SWCNTs) simultaneously allowed for the preparation of hybrid electrodes for 'graphenebased' supercapacitors. The SWCNTs, situated in-between the interlayer space of the graphene sheets as a 'spacer' component, effectively prevent restacking of the graphene that often limits seriously the electrochemical performance of the graphene supercapacitors. The SWCNTs also act as a conductive binder to improve the electrical conduction of the electrode as a whole. The resulting system demonstrated a high specific capacity high specific energy density of $261 \mathrm{~F} \mathrm{~g}^{-}$ ${ }^{1}$ and $123 \mathrm{~W} \mathrm{~h} \mathrm{~kg}^{-1}$ respectively, measured in a two-electrode symmetric system having been obtained in ionic liquid 1-Ethyl-3-methylimidazolium bis(trifluoromethylsulfonyl)imide (EMI-TFSI). ${ }^{13}$

Further to this, with the enhancements in doped graphene oxides, a significant range of potential application of the functionalised graphene have begun to develop and pick up significant interest. For example, Nitrogen-doped reduced graphene oxide (N-rGO), which had been synthesised in the form of 'crumpled' sheets, has been shown to be efficient as the active material in supercapacitor electrodes. A unique deposition method developed was used to manufacture electrodes via 'spray-deposition' of aqueous dispersions of the crumpled nanosheets, resulting in electrodes with a highly modified morphology. The electrochemical charge storage mechanisms of the resulting electrodes were investigated. In this case, a capacitance of up to $9.54 \mathrm{mF} \mathrm{cm} \mathrm{cm}^{-2}$ was exhibited. Despite the choice of units utilised through this study, the results indicate that the N-rGO displays improved performance compared to non-functionalised graphene. ${ }^{14}$

Whilst the typical methodology of drop-casting or spray-coating the electrode substrates is a simple and elegant solution it requires the recoating or depositing of the additive material before each measurement, and may result in some of the additive material being lost from the surface during the measurements. Further to this, these deposition techniques will cause an uncontrollable distribution of the GO upon the electrodes surface resulting in poor 
reproducibility. In order to overcome these issues screen-printed electrodes surfaces are established as effective mass-producible electrochemical sensing platforms that offer versatility in the electrode design. The screen-printing technique can produce vast number of surfaces that exhibit uniform heterogeneous electron transfer kinetics thereby enabling separate electrodes to be used for independent measurements and give consistent/reliable responses.

When graphene has previously been is used in fabrication of supercapacitors devices it has been in the form of rGO. This study is the first time within the literature that GO supercapacitor devices have been produced via the screen-printing technique. By altering the incorporation of GO within the supercapacitor device we seek to explore its effectiveness as a capacitor. 


\section{Experimental method}

GO was synthesized by the oxidation of graphite by the modified Hummers method, shown schematically in Figure 1(A). In a typical batch five grams of graphite flakes were mixed with a mixture of concentrated $\mathrm{H}_{2} \mathrm{SO}_{4}(180 \mathrm{~mL})$ and $\mathrm{H}_{3} \mathrm{PO}_{4}(20 \mathrm{~mL})$. After the mixture stirred for 1 hour, $15 \mathrm{~g}$ of potassium permanganate was added and the mixture was oxidised for 2 hours at $50{ }^{\circ} \mathrm{C}$. Then, $300 \mathrm{~mL}$ of deionized (DI) water and $\mathrm{H}_{2} \mathrm{O}_{2}$ at a ratio of 9:1 was added into the mixture and stirred for 30 mins to remove impurities. Subsequently, the GO washed with DI water several times and collected by centrifugation.

The GO incorporated ink formulations described within the main manuscript were printed using the appropriate stencils by a DEK 248 screen-printing machine (DEK, Weymouth, U.K.), shown schematically Figure 1B. These electrodes have been used extensively in previous studies. A visual description of the SPE/ GO supercapacitor device can be seen in Figure 1(B). In their fabrication; first a carbon-graphite ink formulation (product code C2000802P2; Gwent Electronic Materials Ltd., U.K.) was screen-printed onto a polyester (Autostat, $250 \mu \mathrm{m}$ thickness) flexible film This layer was cured in a fan oven at $60{ }^{\circ} \mathrm{C}$ for 30 minutes. Next, a silver/silver chloride reference electrode was included by screen-printing $\mathrm{Ag} / \mathrm{AgCl}$ paste (product code C2030812P3; Gwent Electronic Materials Ltd., U.K.) onto the polyester substrates and a second curing step was undertaken where the electrodes were cured at $60{ }^{\circ} \mathrm{C}$ for 30 minutes. Finally, a dielectric paste (product code D2070423D5; Gwent Electronic Materials Ltd., U.K.) was then printed onto the polyester substrate to cover the connections. After a final curing at $60{ }^{\circ} \mathrm{C}$ for 30 minutes the SPEs are ready to be used and were connected via an edge connector to ensure a secure electrical connection. The GO was incorporated into the bulk of the SPEs on the basis of the weight percent of $M_{P}$ to $M_{I}$, where $M_{P}$ is the mass of particulate (in this case the GO) and $M_{I}$ is the mass of the ink formulation used in the printing process, i.e. the percentage mass is $M_{\%}=\left(M_{P} / M_{I}\right) \times 100$. The weight percent of $M_{P}$ to $M_{I}$ varied from $2.5,5,7.5$ and $10 \%$, which resulted four separate inks that could subsequently be individually screen-printed on top of the working SPE electrode and cured as described earlier $\left(60{ }^{\circ} \mathrm{C}\right.$ for 30 minutes).

Transmission electron microscopy (TEM) images were obtained using a $200 \mathrm{kV}$ primary beam under conventional bright-field conditions. The GO sample was dispersed onto a holey-carbon film supported on a 300 mesh $\mathrm{Cu}$ TEM grid. Raman Spectroscopy was performed using a 'Renishaw InVia' spectrometer equipped with a confocal microscope ( $\times 50$ objective $)$ and an 
argon laser (514.3 $\mathrm{nm}$ excitation). Measurements were performed at a very low laser power level $(0.8 \mathrm{~mW})$ to avoid any heating effects. X-ray diffraction (XRD) was performed using an “X'pert powder PANalytical" model with a copper source of K $\alpha$ radiation (of $1.54 \AA$ ) and $\mathrm{K} \beta$ radiation (of $1.39 \AA$ ), using a thin sheet of nickel with an absorption edge of $1.49 \AA$ to absorb $\mathrm{K} \beta$ radiation. A reflection transmission spinner stage $(15 \mathrm{rpm})$ was implemented to hold the commercially sourced GO nano-powder. The range was set between 5 and $802 \theta$ in correspondence with literature ranges ${ }^{15}$. Additionally, to ensure well defined peaks an exposure of 50 seconds per $2 \theta$ step was implemented with a size of $0.013^{\circ}$. The $\mathrm{x}$-ray photoelectron spectroscopy (XPS) data was acquired using a bespoke ultra-high vacuum system fitted with a Specs GmbH Focus 500 monochromated Al Ka X-ray source, Specs GmbH Phoibos $150 \mathrm{~mm}$ mean radius hemispherical analyser with 9-channeltron detection, and a Specs GmbH FG20 charge neutralising electron gun ${ }^{16}$. Survey spectra were acquired over the binding energy range $1100-0 \mathrm{eV}$ using a pass energy of $50 \mathrm{eV}$ and high resolution scans were made over the $\mathrm{C} 1 \mathrm{~s}$ and $\mathrm{O} 1 \mathrm{~s}$ lines using a pass energy of $20 \mathrm{eV}$. Under these conditions the full width at half maximum of the $\mathrm{Ag} 3 \mathrm{~d} 5 / 2$ reference line is ca. $0.7 \mathrm{eV}$. In each case, the analysis was an area-average over a region approximately $1.4 \mathrm{~mm}$ in diameter on the sample surface, using the $7 \mathrm{~mm}$ diameter aperture and lens magnification of $\times 5$. The energy scale of the instrument is calibrated according to ISO 15472, and the intensity scale is calibrated using an in-house method traceable to the UK National Physical Laboratory ${ }^{17}$. Data were quantified using Scofield cross sections corrected for the energy dependencies of the electron attenuation lengths and the instrument transmission ${ }^{18}$. Data interpretation was carried out using CasaXPS software v2.3.16 ${ }^{19}$.

The capacitive characterisations of the Graphene Oxide enhanced electrodes (GOEEs) was carried out by galvanostatic charge-discharge with the fixed currents ranging from 0.5 to 50 $\mu \mathrm{A}$, from 0 to $1 \mathrm{~V}$. The capacitive characterisation is carried out in three different electrolytes, 1.0 $\mathrm{M} \mathrm{H}_{2} \mathrm{SO}_{4}, 6.0 \mathrm{M} \mathrm{KOH}$, and a non-aqueous solution of Room Temperature Ionic Liquid (RTIL) 1-butyl-3-methylimidazolium hexafluorophosphate, $\left[\mathrm{C}_{4} \mathrm{MIM}\right]\left[\mathrm{PF}_{6}\right]$. All chemicals used were of analytical grade, were used as received without any further purification, and were obtained from Sigma-Aldrich. All solutions were prepared, where appropriate, with deionised water of resistivity not less than $18.2 \mathrm{M} \Omega \mathrm{cm}$.

Capacitance and voltammetric measurements were performed using an 'Autolab - $\mu$ Autolab Type III' (Metrohm Autolab, The Netherlands) potentiostat. All measurement s reported herein were performed utilising a symmetrical GO two-electrode system. The capacitance, $C_{\text {total }}$ of 
the supercapacitor is evaluated from the slope of the charge/discharge cycles using the following:

$$
C_{\text {total }}=\frac{I}{(d V / d t)}
$$

where $I$ is the current applied, $V$ is the potential measured over time, $t$.

Scanning electron microscope (SEM) images and surface element analysis were obtained with a JEOL JSM 5600LV model having energy-dispersive X-ray microanalysis package. 


\section{Results and Discussion}

Initially it was essential to perform a full physicochemical characterisation of the GO powder in order to ascertain its quality/properties prior to it being incorporated into the screen-printed electrode. Raman spectroscopy, TEM, XPS and XRD analysis were all conducted.

The atomic structural composition of the GO sample is confirmed via XRD in Figure 2(A), in which a characteristic 'sharp' peak is evident at $2 \theta=11.5^{\circ}$, corresponding to the $(001)$ diffraction peak of disordered GO. Figure 2(B) displays TEM of the GO platelets and indicates that they have an average particle size (lateral width) of ca. 300 to $600 \mathrm{~nm}$. Next, XPS analysis was performed to determine the GOs elemental composition with Figure 2(D) showing the gathered survey spectra and ESI Figure 1 displaying the individual spectra for the $\mathrm{C}$ and $\mathrm{O}$ regions. The GO was observed to contain $66.8 \%$ carbon and $28.6 \%$ oxygen with trace amounts of nitrogen, sulphur and chlorine which are likely merely contaminants. Specifically, groups corresponding to graphitic $\mathrm{C}-\mathrm{C}$ bonding in addition to $\mathrm{C}-\mathrm{O}$ or $\mathrm{C}-\mathrm{O}-\mathrm{C}$ bonds $(47.21 \%, 286.7$ $\mathrm{eV})$ and $\mathrm{C}=\mathrm{O}$ or $\mathrm{COO}(7.94 \%, 288.4 \mathrm{eV})$ bonds where characteristically present, which is in excellent agreement with previous literature reports regarding GO. ${ }^{1,1,13}$ ESI Table 1 shows the elemental composition of a bare/unmodified SPE and the GO-SPEs, it is clear from inspection of this table that there is a increase in the oxygen present on each platforms surface associated with an increase in the GO present within the supercapacitor device. Finally, Raman spectroscopy was utilised to confirm the presence of GO by structural characterisation, the obtained spectra can be viewed in Figure 2(D) and displays the D and G vibrational band peaks at $c a .1350$ and $1590 \mathrm{~cm}^{-1}$ respectively that are typically characteristic of GO. The combination of surface and physicochemical analysis presented above and expanded upon with the SI confirm that the GO herein utilised is of a high quality and purity.

The capacitive properties of the GO-SPE are first considered in an aqueous solution, being compared and benchmarked to a standard commercially available graphitic SPE. It should be noted that in each of the experiments a symmetric arrangement is utilised, ensuring that both the working and counter electrodes are of the same material, and same composition. The electrodes are connected and submerged into the electrolyte in an orthodox cell. The capacitive performance is evaluated by means of galvanostatic charge and discharge cycling. The systems are charged at fixed currents from 0.5 to $50 \mu \mathrm{A}$, to a fixed potential window 0 to $1 \mathrm{~V}$ and vice versa for discharge. 
In order to evaluate the capacitive performance of the structures each sample is subjected to charging currents from $0.5 \mu \mathrm{A}\left(1.12 \mathrm{~A} \mathrm{~g}^{-1}\right)$ to $50 \mu \mathrm{A}\left(11.2 \mathrm{~A} \mathrm{~g}^{-1}\right)$. The charge/discharge properties of the GO-SPEs are shown in Figure $4 \mathrm{~A}$, which shows the influence of the increase in current and Figure $4 \mathrm{~B}$ which shows the influence of the increase in GO content. In Figure $4 \mathrm{~A}$ the $5 \% \mathrm{GO}$ enhanced screen printed electrode is cycled in $3.0 \mathrm{M} \mathrm{KOH}$. The chargedischarge profile demonstrates near linear growth over the potential window from 0 to $1.0 \mathrm{~V}$, over times from 139.3 to $276.3 \mathrm{~s}$, corresponding to charging current densities from 11.2 down to $1.12 \mathrm{~A} \mathrm{~g}^{-1}$ respectively. It can be seen here that the increase in GO content has a significant impact on the capacitive charge discharge properties of the electrodes, significantly reducing the capacitance.

For each electrode composition and electrolyte, the galvanostatic charge discharge is again carried out at currents from 0.5 to $50 \mu \mathrm{A}$. Over this range a typical SPE, with $0 \% w t$. of GO, demonstrates a capacitance in the $3.0 \mathrm{M} \mathrm{KOH}$ aqueous electrolyte ranging from $59 \mu \mathrm{F}$ down to $28 \mu \mathrm{F}$ (equating to roughly 0.21 to $0.10 \mathrm{~F} \mathrm{~g} \mathrm{~g}^{-1}$ ) for the respective current range. The GO enhanced SPEs are tested in the same electrolytes and same conditions, demonstrating capacitances from 76 to $134 \mu \mathrm{F}$ (equating to 134 down to $50 \mathrm{~F} \mathrm{~g}^{-1}$ respectively) in $\mathrm{KOH}$ at a charging current of $0.5 \mu \mathrm{A}$, over an increase in graphene content from 2.5 to $10 \% \mathrm{wt}$. The addition of the GO to the electrode ink increases the capacitive performance and capabilities by a factor of more than 500. Further to this, the performance in another commonly used aqueous electrolyte, $1.0 \mathrm{M} \mathrm{H}_{2} \mathrm{SO}_{4}$, is also investigated for the same current ranges. Under these conditions the SPE, which is again utilised to benchmark the impact of the GO, demonstrate a capacitance of $64 \mu \mathrm{F}$ (equating to $0.24 \mathrm{~F} \mathrm{~g}^{-1}$ ) at a current of $0.5 \mu \mathrm{A}$. The enhanced GO-SPEs, demonstrate further improvement in capacitance, from 69 to $98 \mu \mathrm{F}$ for 2.5 to $10 \% \mathrm{GO}$, for the same charging current (equating to 121.69 to $36.58 \mathrm{Fg}^{-1}$ ). Additionally, the performance in a Room Temperature Ionic liquid (RTIL) 1-butyl-3-methylimidazolium hexafluorophosphate, $\left[\mathrm{C}_{4} \mathrm{MIM}\right]\left[\mathrm{PF}_{6}\right]$, is utilised to fully understand the potential of $\mathrm{GO}$ as an electrode modification. Utilising this electrolyte, a significant enhancement in the capacitive performance is once again observed; demonstrating a competitively high specific capacitance of $423 \mathrm{Fg}^{-1}$ across the electrode range. This is a notable improvement upon the standard electrode, which demonstrates a capacitance of $4.87 \mathrm{Fg}^{-1}$. As a side note, the specific capactience of the GO is greater than previous work on graphene/polyaniline nanocomposites $(257 \mathrm{~F} / \mathrm{g}){ }^{20}$

It should be noted that there is a negative correlation between the weight of the GO and the specific capacitance of the electrodes, as highlighted by Table 1, which summarises the 
capacitance of the electrodes in each of the electrolytes at a fixed current of $0.5 \mu \mathrm{A}$, and figure $4 \mathrm{C}$, which illustrates the capacitance and specific capacitance of the electrodes in terms of the $\% \mathrm{wt}$, of graphene content. This is indicative of the deviation in the incremental additive mass and the increase in the GO content, in that the mass increase is more influential than the improvement in performance; perhaps highlighting the requirement the importance of capacitive performance alone. In the table and figure respectively it can be seen that there is linear improvement in the capacitance of the systems with the increase in GO content. It should be noted that manufacturing an electrode with a $\%$ wt. of GO greater than $10 \%$ is impractical as a result of the high surface energy of the GO nanosheets, generating a wicking or drying effect on the ink. The resulting ink when higher GO \% wt. is utilised is too dry and particulate to screen print.

The enhancement of the screen-printing ink with GO nanosheets demonstrates conclusively that the application of GO in energy storage electrodes can significantly improve the capacitive performance of the electrode material. Further to this, the performance of the resulting electrodes is highly competitive, as summarised by Figure 6. A Ragone plot of the performance of the enhanced graphene electrodes and some of the most competitive similar materials. The results here are compare to Poly (Ionic Liquid) - Modified reduced GO electrodes; ${ }^{21}$ a symmetrical graphene based supercapacitor; ${ }^{22}$ a symmetrical supercapacitor using reduced GO, modified with ruthenium oxide and polyaniline, ${ }^{23}$ An asymmetric supercapacitors of graphene and $\mathrm{MnO}_{2}$ nanowires; a manganese oxide activated carbon hybrid capacitor; ${ }^{24}$ and a supercapacitor utilising metal oxide nanowires/carbon nanotube thin film electrodes. ${ }^{4}$ The performance of the GO-SPEs is shown to demonstrate a power ranging from 1.23 to $3.08 \mathrm{~kW}$ $\mathrm{kg}^{-1}$ in $\mathrm{H}_{2} \mathrm{SO}_{4}$, from 1.47 to $2.97 \mathrm{~kW} \mathrm{~kg}^{-1}$ in $\mathrm{KOH}$ and 4.50 up to 13.99 in $\mathrm{kW} \mathrm{kg}^{-1}$ in $\left[\mathrm{C}_{4} \mathrm{MIM}\right]\left[\mathrm{PF}_{6}\right]$, which is shown to be highly competitive in the Ragone plot. Further to this this application of the integrated screen printing ink also includes the material in the conduction paths along the electrodes, further indicative of the high performance of the enhanced electrode material. 


\section{Conclusion}

Over the last decade interest in graphene has significantly overshadowed the research into alternative material research, resulting in simpler alternatives being overlooked. Graphene oxide, more-often-than-not, is a product manufactured en-route to the manufacture of graphene or reduced graphene oxide (rGO). The application of graphene oxide as an advanced material for energy storage applications could significantly reduce the cost and improve the yield of manufacturing high performance electrodes. As such, this is the first study of utilising graphene oxide as a functional material for the enhancement of electrodes in a supercapacitor device. A screen-printing ink is enhanced with the integration of functionalised graphene to provide a contextual analysis of the impact of GO in supercapacitor electrodes. The addition of the GO to the electrode ink increases the capacitive performance and capabilities by a factor of more than 500. The electrodes are investigated in $\mathrm{KOH}$ and $\mathrm{H}_{2} \mathrm{SO}_{4}$ aqueous electrolytes. The performance of the GO-SPEs is shown to demonstrate a power ranging from 1.23 to $3.08 \mathrm{~kW}$ $\mathrm{kg}^{-1}$ in $\mathrm{H}_{2} \mathrm{SO}_{4}$, from 1.47 to $2.97 \mathrm{~kW} \mathrm{~kg}^{-1}$ in $\mathrm{KOH}$ and 4.50 up to 13.99 in $\mathrm{kW} \mathrm{kg}^{-1}$ in $\left[\mathrm{C}_{4} \mathrm{MIM}\right]\left[\mathrm{PF}_{6}\right]$, which is shown to be highly competitive within the field of supercapacitor research. 


\section{Acknowledgements}

Funding from the Engineering and Physical Sciences Research Council (Reference: EP/N001877/1) and a British Council Institutional Grant Link (No. 172726574) is acknowledged. 
Table 1. A summary of the capacitive performance of the GO and a typical graphite supercapacitor, indicating the impact and improvement in performance with the application of the graphene oxide in electrodes for energy storage applications. It is shown that the increase in graphene oxide there is a significant and notable increase in the capacitive performance in all electrolytes.

\begin{tabular}{|c|c|c|c|c|c|c|}
\hline \multirow[b]{2}{*}{$\%$ wt. } & \multicolumn{2}{|c|}{$3 \mathrm{M} \mathrm{KOH}$} & \multicolumn{2}{|c|}{$1.0 \mathrm{M} \mathrm{H}_{2} \mathrm{SO}_{4}$} & \multicolumn{2}{|c|}{$\left[\mathrm{C}_{4} \mathrm{MIM}\right]\left[\mathrm{PF}_{6}\right]$} \\
\hline & $\mathrm{C}(\boldsymbol{\mu F})$ & $C_{s}\left(F g^{-1}\right)$ & $\mathrm{C}(\boldsymbol{\mu F})$ & $C_{s}\left(F g^{-1}\right)$ & $C(\mu F)$ & $\mathrm{C}_{\mathrm{s}}\left(\mathrm{F} \mathrm{g}^{-1}\right)$ \\
\hline 0 & 56 & 0.21 & 64 & 0.24 & 130 & 4.87 \\
\hline 2.5 & 76 & 134.03 & 69 & 121.69 & 240 & 423.28 \\
\hline 5 & 89 & 101.54 & 74 & 84.42 & 326 & 371.93 \\
\hline 7.5 & 102 & 65.09 & 86 & 54.88 & 452 & 288.44 \\
\hline 10 & 134 & 50.02 & 98 & 36.58 & 562 & 209.78 \\
\hline
\end{tabular}


Figure 1. (A) Schematic of the formation of the GO nanoflakes by means of the Hummers methodology; (B) schematic illustration of the process of manufacturing the modified GO enhanced supercapacitor device. (C) and schematic illustration of the cell set up.

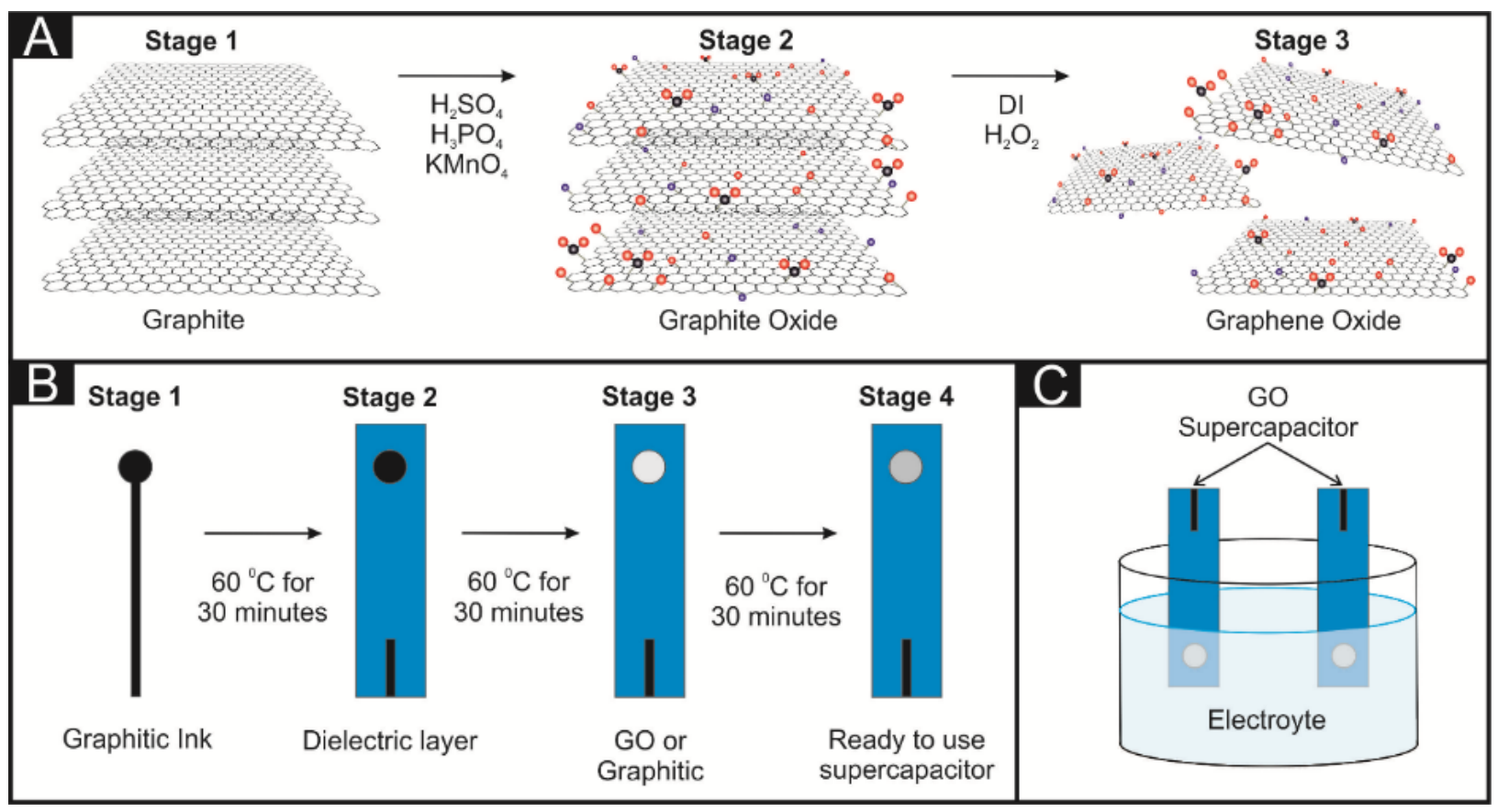


Figure 2. Characterisation of the GO; TEM images of GO nanosheets (A, Scale bar: $500 \mathrm{~nm}$ ), (B, Scale bar: $100 \mathrm{~nm}$ ) used in the fabrication of the GO supercapacitor devices; (C) XPS survey spectra and; (D) Raman spectra of GO deposited onto a silicon wafer between 100 and $3400 \mathrm{~cm}^{-1}$.

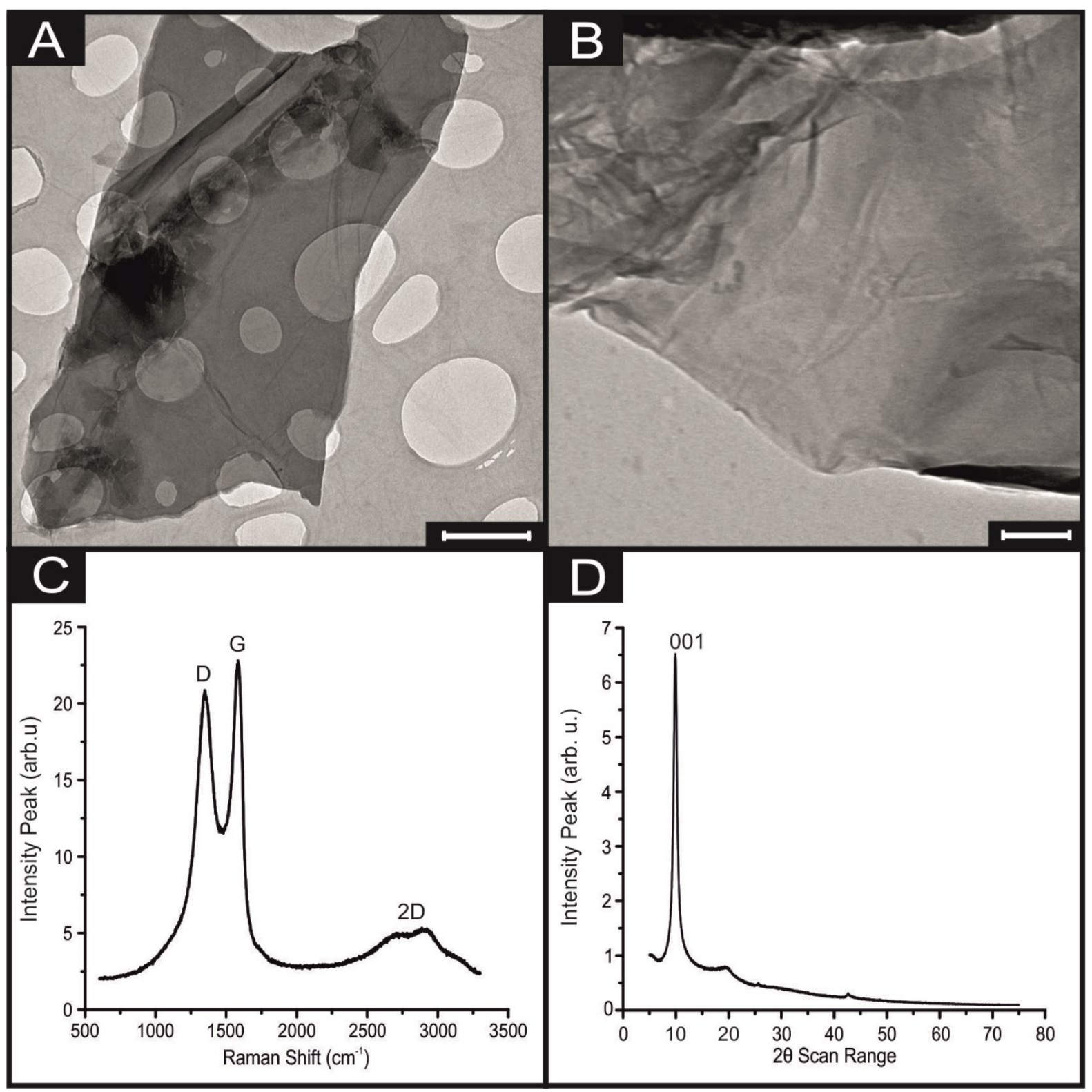


Figure 3. High-resolution XPS spectra of $\mathrm{C}$ and $\mathrm{O}$ regions of the $\mathrm{GO}$ utilised in the formation of the GO supercapacitor device (A and B respectively). (C) XPS survey spectra.

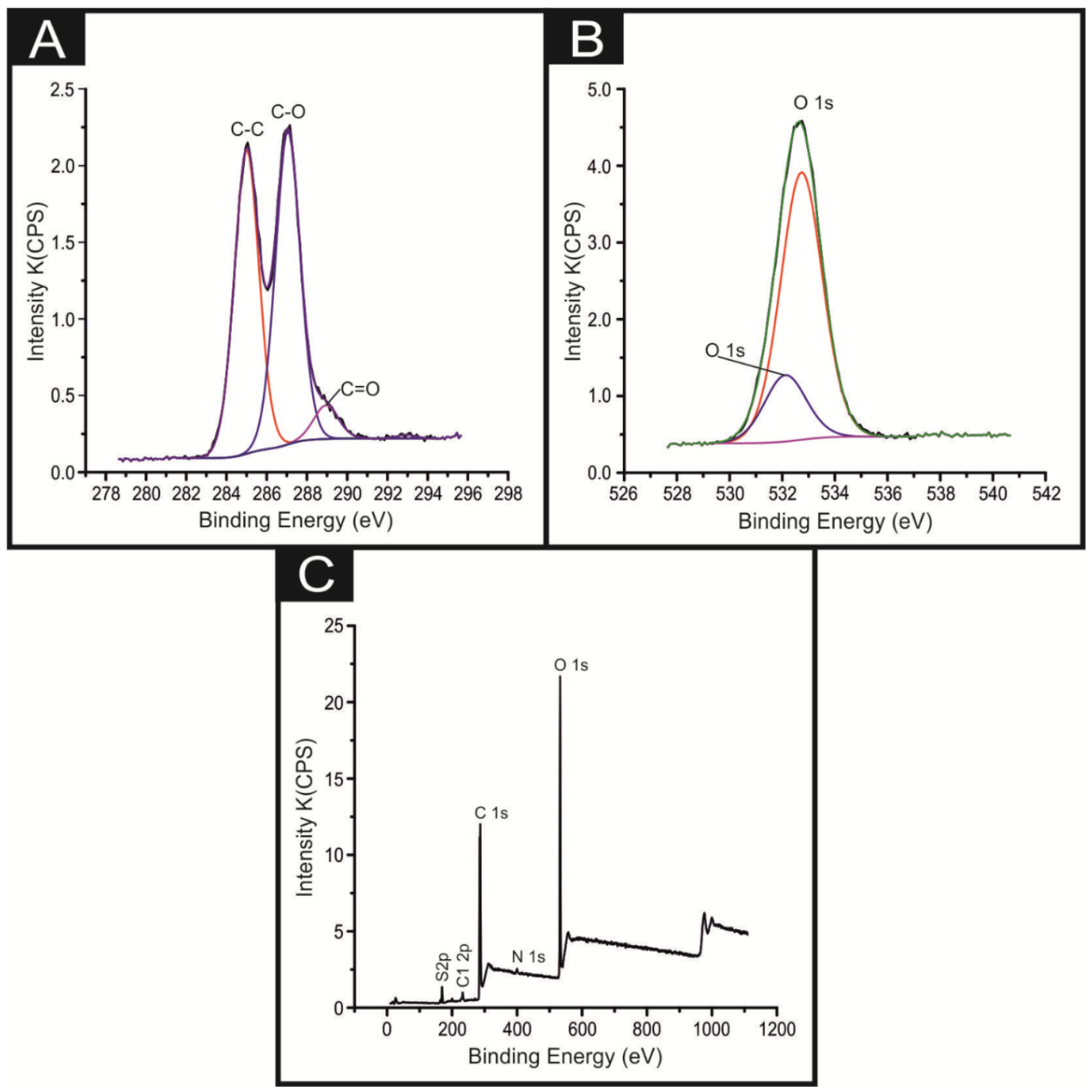


Figure 4. Capacitance behaviour of the GO supercapacitor and a graphite supercapacitor: A) depicts the charge-discharge behaviour of $2.5 \% \mathrm{wt}$. GO for increasing charging and discharge currents from $0.5 \mu \mathrm{A}\left(1.12 \mathrm{~A} \mathrm{~g}^{-1}\right)$ to $50 \mu \mathrm{A}\left(11.2 \mathrm{Ag}^{-1}\right)$. B) The charge-discharge behaviour at a fixed current for increasing graphene oxide content. C) The capacitive performance of the graphene oz

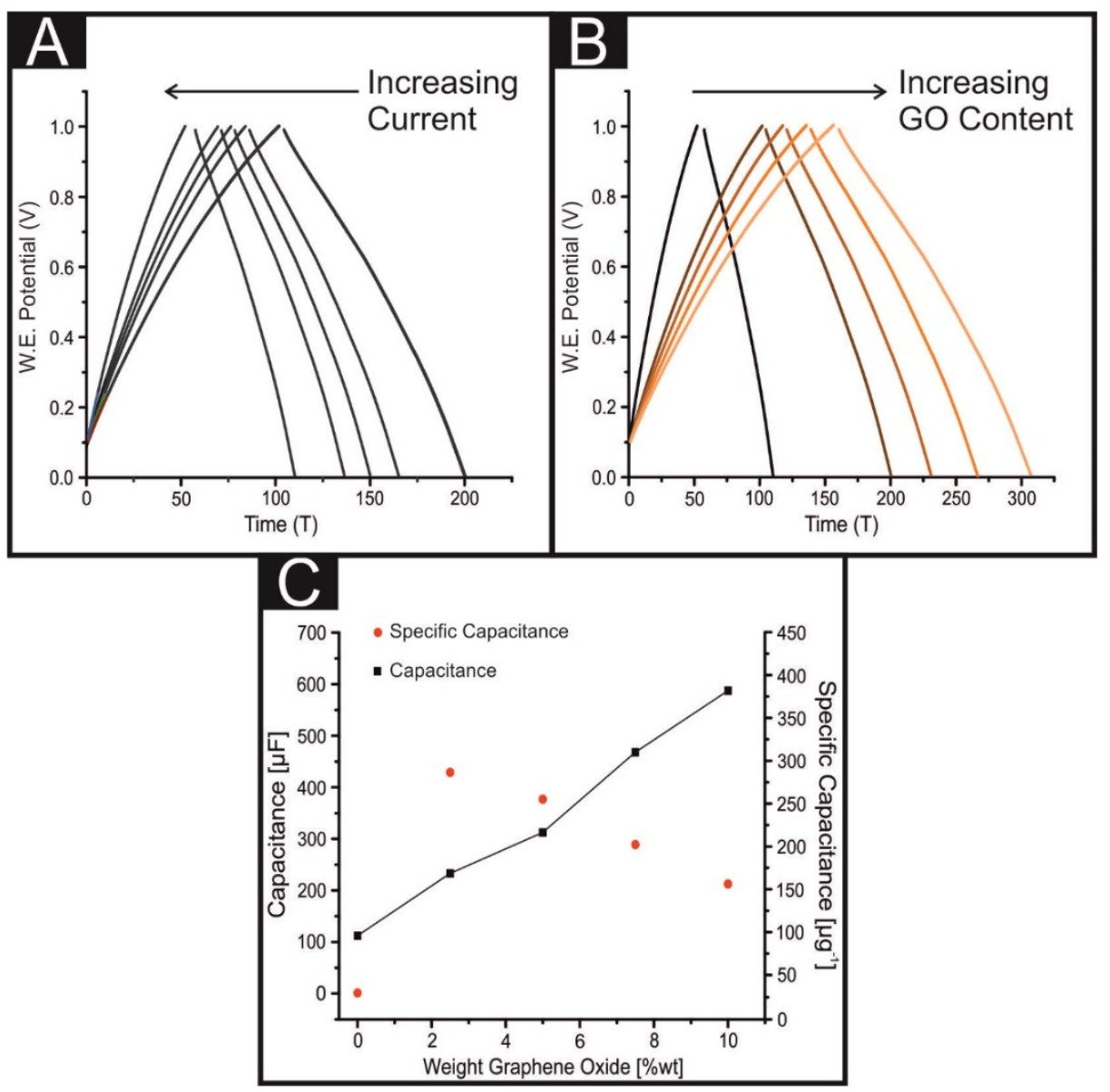


Figure 5. The capacitive performance of the graphene-oxide enhanced supercapacitors in $\left[\mathrm{C}_{4} \mathrm{MIM}\right]\left[\mathrm{PF}_{6}\right](\mathrm{A})$ and the $2.5 \% \mathrm{GO}$ in the tested electrolytes $(\mathrm{B})$ over the investigated current ranges.

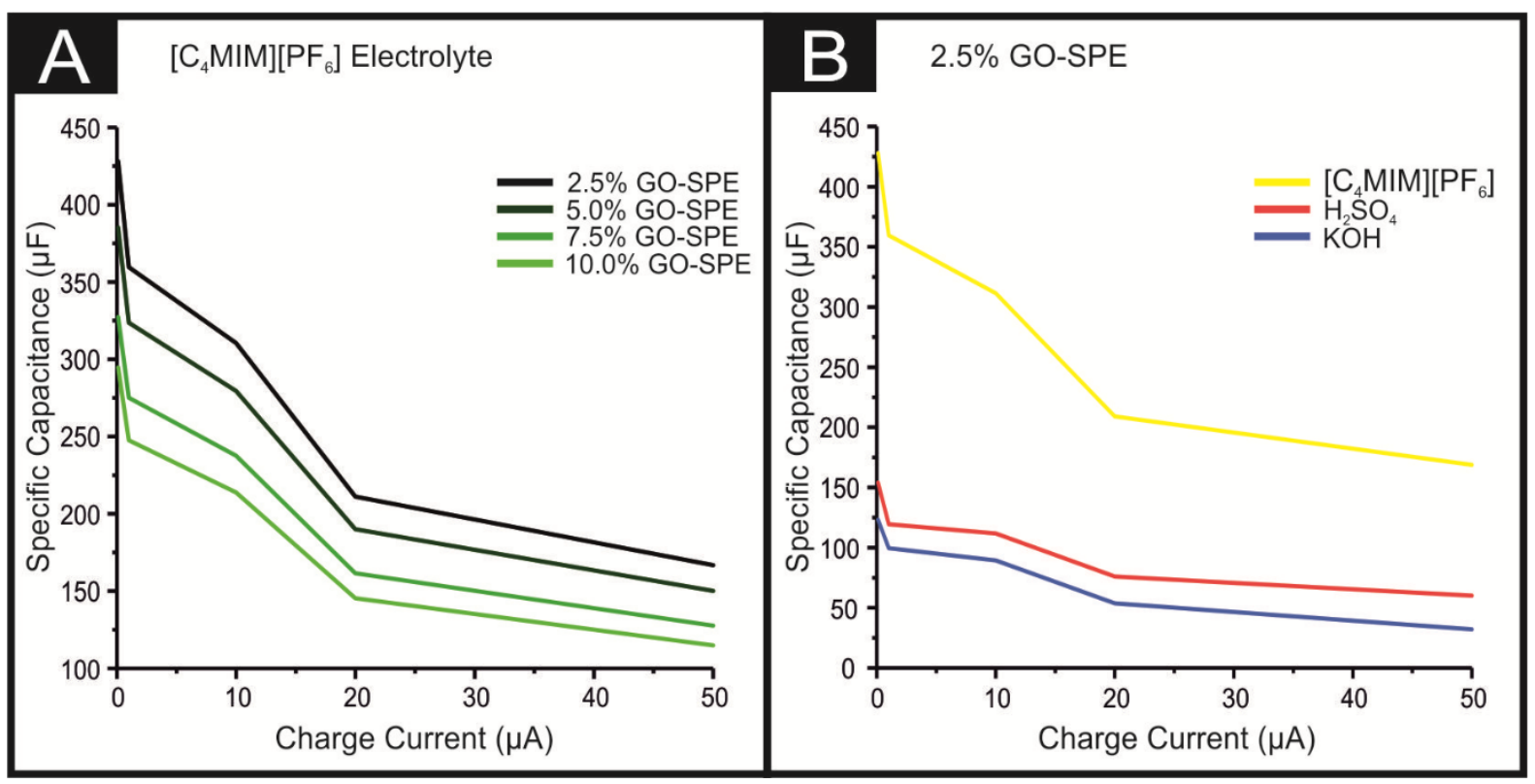


Figure 6. The Ragone plot, showing the Energy and Power densities of the GO in context to other similar materials and studies. The resulting performance demonstrates values at the higher end of both the energy and power in the spectrum normally considered the norm for supercapacitors. The results here are compare to Poly (Ionic Liquid) - Modified reduced graphene oxide electrodes: ${ }^{21}$ a symmetrical graphene based supercapacitor, ${ }^{22}$ a symmetrical supercapacitor using reduced graphene oxide, modified with ruthenium oxide and polyaniline ${ }^{23}$; An asymmetric supercapacitors of graphene and $\mathrm{MnO}_{2}$ nanowires; a manganese oxide activated carbon hybrid capacitor; ${ }^{24}$ and a supercapacitor utilising metal oxide nanowires/carbon nanotube thin film electrodes. ${ }^{4}$ The samples tested in this study are highlighted as triangles, whilst circles represent performance of similar devices from literature.

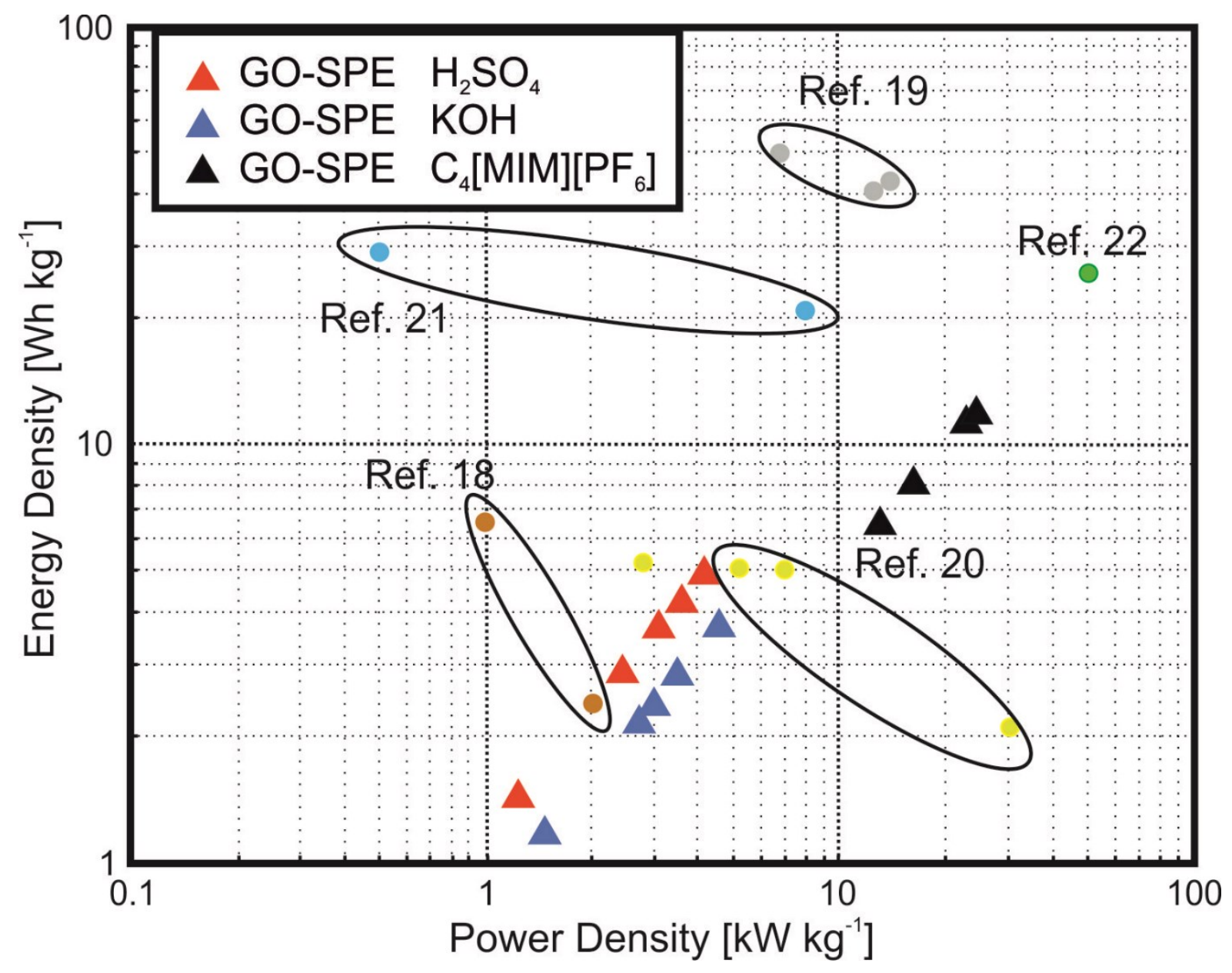




\section{References}

1. $\quad$ M. J. Allen, V. C. Tung and R. B. Kaner, Chem. Rev., 2010, 110, 132-145.

2. $\quad$ M. P. Down, C. W. Foster, X. Ji and C. E. Banks, RSC Adv., 2016, 6, 81130-81141.

3. B. G. Choi, M. Yang, W. H. Hong, J. W. Choi and Y. S. Huh, ACS Nano, 2012, 6, 40204028 .

4. $\quad$ P.-C. Chen, G. Shen, Y. Shi, H. Chen and C. Zhou, ACS Nano, 2010, 4, 4403-4411.

5. S. Boukhalfa, K. Evanoff and G. Yushin, Energy Environ Sci, 2012, 5, 6872-6879.

6. D. A. C. Brownson, C. S. Figueiredo-Filho, X. Ji, M. Gomez-Mingot, J. Iniesta, O. FatibelloFilho, D. K. Kampouris and C. E. Banks, J. Mater. Chem. A, 2013, 1, 5962-5972.

7. M. P. Down, L. Jiang and J. W. McBride, Appl. Phys. Lett., 2015, 107, 071901.

8. C.-M. Chen, Q. Zhang, C.-H. Huang, X.-C. Zhao, B.-S. Zhang, Q.-Q. Kong, M.-Z. Wang, Y.G. Yang, R. Cai and D. S. Su, Chem. Commun., 2012, 48, 7149-7151.

9. T. J. Davies, M. E. Hyde and R. G. Compton, Angew. Chem. Int. Ed., 2005, 44, 5121-5126.

10. A. K. Geim, Science, 2009, 324, 1530-1534.

11. P. Binh, L. Thanh, M. Xuan, M. T. Thuy and P. Tot, Adv. Nat. Sci.: Nanosci., 2016, 7, 015016.

12. J. Xia, F. Chen, J. Li and N. Tao, Nat Nano, 2009, 4, 505-509.

13. F. Zhang, J. Tang, N. Shinya and L.-C. Qin, Chem. Phys. Lett., 2013, 584, 124-129.

14. Y. Zou, I. A. Kinloch and R. A. W. Dryfe, J. Mater. Chem. A, 2014, 2, 19495-19499.

15. Z. Lei, S. Xu and P. Wu, Phys. Chem. Chem. Phys., 2016, 18, 70-74.

16. C. Tsai, K. Chan, F. Abild-Pedersen and J. K. Norskov, Phys. Chem. Chem. Phys., 2014, 16, 13156-13164.

17. M. P. Seah and S. J. Spencer, J. Electron. Spectrosc. Relat. Phenom., 2006, 151, 178-181.

18. J. H. Scofield, J. Electron. Spectrosc. Relat. Phenom., 1976, 8, 129-137.

19. K. Liu, L. Zhang, T. Cao, C. Jin, D. Qiu, Q. Zhou, A. Zettl, P. Yang, S. G. Louie and F. Wang, Nat. Commun., 2014, 5, 4966.

20. Z.-F. Li, H. Zhang, Q. Liu, L. Sun, L. Stanciu and J. Xie, ACS Appl. Mater. Interfaces, 2013, 5, 2685-2691.

21. T. Y. Kim, H. W. Lee, M. Stoller, D. R. Dreyer, C. W. Bielawski, R. S. Ruoff and K. S. Suh, ACS Nano, 2011, 5, 436-442.

22. J. Zhang, J. Jiang, H. Li and X. S. Zhao, Energy Environ. Sci., 2011, 4, 4009-4015.

23. Z.-S. Wu, W. Ren, D.-W. Wang, F. Li, B. Liu and H.-M. Cheng, ACS Nano, 2010, 4, 58355842 .

24. M. S. Hong, S. H. Lee and S. W. Kim, Electrochem. Solid-State Lett., 2002, 5, A227-A230. 


\section{Electronic Supplementary Information:}

\section{Fabrication of Graphene Oxide Supercapacitor Devices}

Michael P. Down ${ }^{1,2}$, Samuel J. Rowley-Neale ${ }^{1,2}$, Graham C. Smith ${ }^{3}$ and Craig E. Banks ${ }^{1,2 *}$

${ }^{1}$ :Faculty of Science and Engineering, Manchester Metropolitan University, Chester Street, Manchester, M1 5GD, UK

${ }^{2}:$ Manchester Fuel Cell Innovation Centre, Manchester Metropolitan University, Chester Street, Manchester M1 5GD, UK.

${ }^{3}$ : Faculty of Science and Engineering, Department of Natural Sciences, University of Chester, Thornton Science Park, Pool Lane, Ince, Chester CH2 4NU, UK.

*To whom correspondence should be addressed.

Email: c.banks@mmu.ac.uk; Tel: ++(0)1612471196; Fax: ++(0)1612476831

Website: www.craigbanksresearch.com 
ESI Table 1. XPS elemental analysis of a bare/unmodified SPE and a 2.5, 5, 7.5 and $10 \%$ GOSPE.

\begin{tabular}{lrrrrr}
\hline Element & Graphite SPE & $\mathbf{2 . 5 \%}$ GO-SPE & $\mathbf{5 \%}$ GO-SPE & $\mathbf{7 . 5 \%}$ GO-SPE & $\mathbf{1 0 \%}$ GO-SPE \\
\hline C 1s & 79.72 & 77.96 & 78.24 & 76.47 & 76.31 \\
O 1s & 11.37 & 14 & 13.36 & 15.05 & 16.07 \\
Cl 2p & 7.69 & 6.79 & 6.97 & 6.76 & 5.29 \\
Si 2p & 0.77 & 0.89 & 0.56 & 0.82 & 0.54 \\
Al 2p & 0.46 & 0.36 & 0.45 & 0.26 & 0.46 \\
N 1s & & & 0.42 & 0.33 & 0.71 \\
S 2p & & & & 0.32 & 0.62 \\
\hline
\end{tabular}


ESI Figure 1. SEM images of the graphite and GO electrode surfaces in the supercapacitor device show little variation in the surface morphology of the surfaces with variation in GO content. Given this, it is apparent that the dominating influence of the morphology of the electrodes is in fact the carbon ink. This indicates that the improvement in the performance is a result of physicochemical properties of the graphene oxide, and not a result of any morphological differences induced by the addition of the GO.

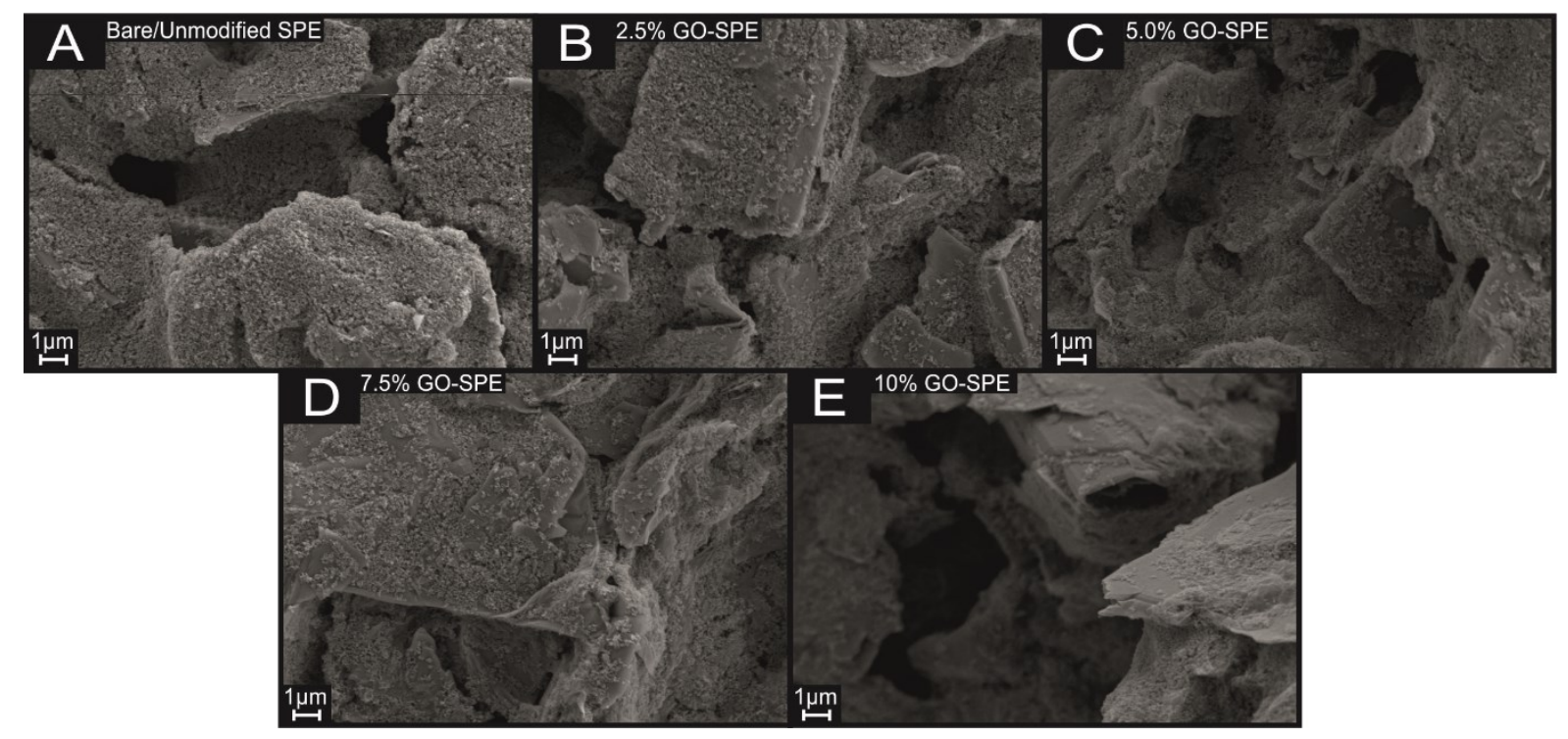


ESI Figure 2. Capacitance retention of the $10 \% \mathrm{GO}$ device when compared to a graphite device over 5000 cycles, with a switching period of 30 s and a charging current of $50 \mu \mathrm{A}$.

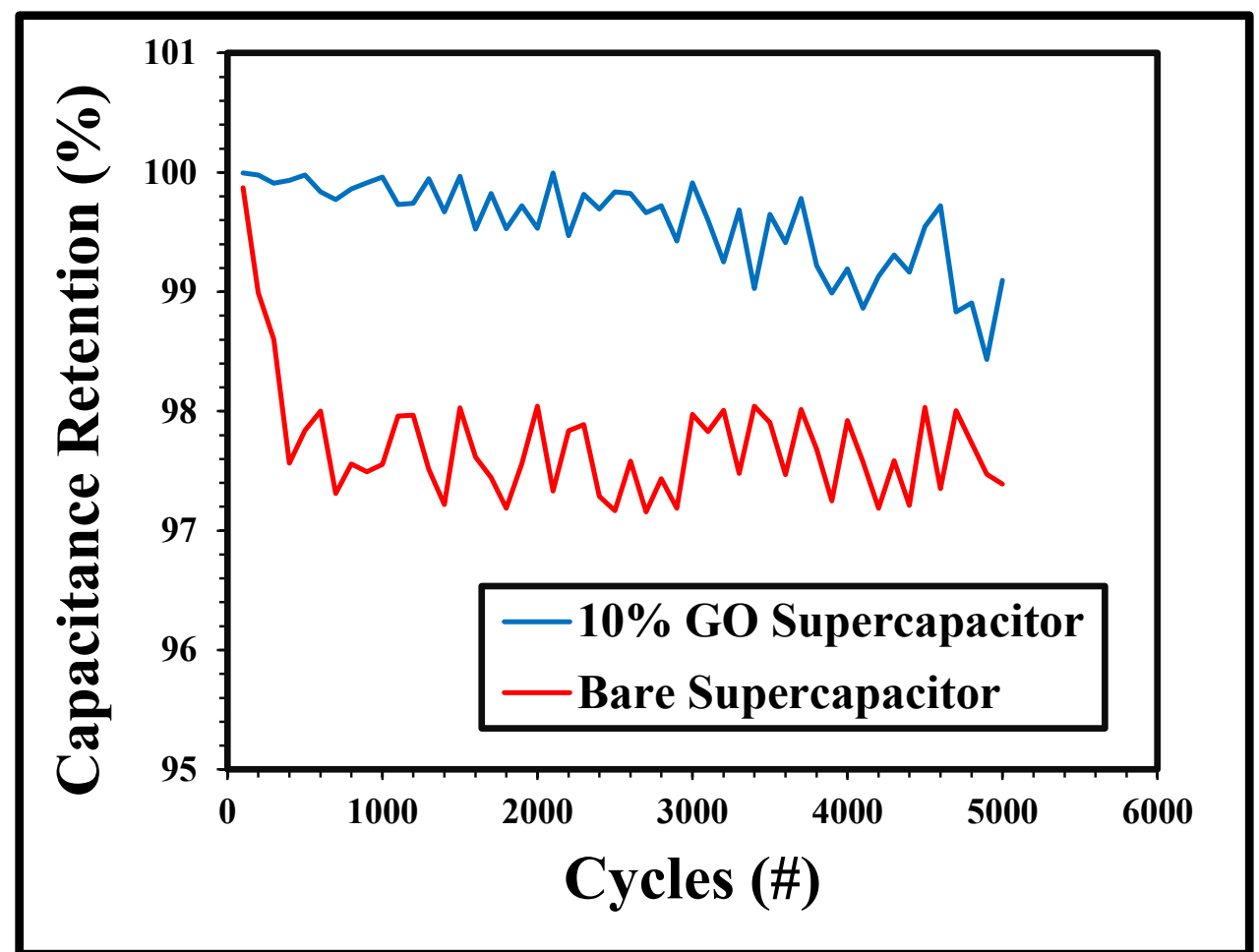

